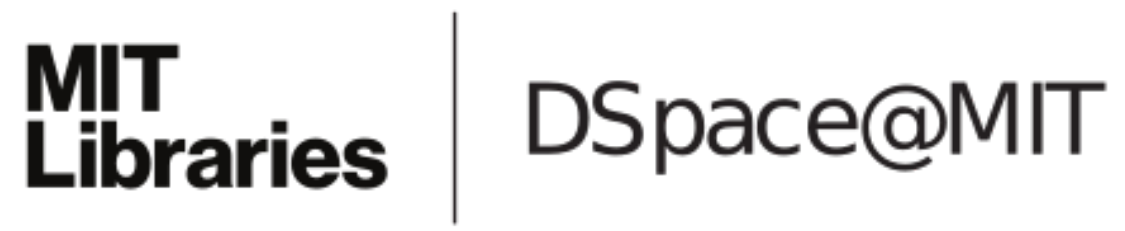

\author{
MIT Open Access Articles
}

Quantifying the nanomachinery of the nanoparticle-biomolecule interface

The MIT Faculty has made this article openly available. Please share how this access benefits you. Your story matters.

Citation: de Puig, Helena et al. "Quantifying the Nanomachinery of the NanoparticleBiomolecule Interface." Small (2011) : v. 7, issue 17, p. 2477-2484, Sept. 2011. Copyright @ 2011 WILEY-VCH Verlag GmbH \& Co.

As Published: http://dx.doi.org/10.1002/smll.201100530

Publisher: Wiley-VCH

Persistent URL: http://hdl.handle.net/1721.1/65656

Version: Author's final manuscript: final author's manuscript post peer review, without publisher's formatting or copy editing

Terms of use: Creative Commons Attribution-Noncommercial-Share Alike 3.0 


\title{
Quantifying the nanomachinery of the nanoparticle-biomolecule interface
}

1,4, ${ }^{*}$ Helena de Puig Guixé, ${ }^{3,}$ Stefania Federici, ${ }^{1}$ Salmaan H. Baxamusa, ${ }^{3, \#}$ Paolo Bergese, and

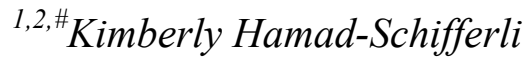

${ }^{1}$ Department of Biological Engineering, ${ }^{2}$ Department of Mechanical Engineering, Massachusetts Institute of Technology, 77 Massachusetts Ave, Cambridge, MA, 02139, US

${ }^{3}$ Chemistry for Technologies Laboratory and INSTM, University of Brescia, Via Branze, 38, 25123 Brescia, Italy

${ }^{4}$ Institut Quimic de Sarrià, Universitat Ramon Llull, Via Augusta 390, 08017 Barcelona, Spain

*These authors equally contributed to the work.

${ }^{\#}$ Corresponding authors

Keywords: nanorod, nanomachinery, surface work, self-assembly, thrombin, nanoparticlebiomolecule interface

\begin{abstract}
We present a study of the nanomechanical phenomena experienced by nanoparticle-conjugated biomolecules. We develop a thermodynamic framework to describe binding of thrombin binding aptamer (TBA) to thrombin when the TBA is conjugated to nanorods. Binding results in nanorod aggregation (viz. directed self-assembly) which is detectable by absorption spectroscopy. The analysis introduces the energy of aggregation, separating it into TBA-thrombin recognition and surface work contributions. Consequently, we demonstrate that self-assembly is driven by the interplay of surface work and thrombin-TBA recognition. We show that surface work is $\sim-10$
\end{abstract}


$\mathrm{kJ} / \mathrm{mol}$ and results from accumulation of in-plane molecular forces of $\mathrm{pN}$ magnitude and lifetime $<1$ second, which arise from TBA nanoscale rearrangements fuelled by thrombin-directed NR aggregation. The obtained surface work can map aggregation regimes as a function of different nanoparticle surface conditions. Also, the thermodynamic treatment can be used to obtain quantitative information on surface effects impacting biomolecules on nanoparticle surfaces.

\section{Introduction}

Biomolecular machines have inspired engineers for their ability to perform mechanical work in a way that is rapid, specific, and with efficiencies that surpass artificial systems. Many have sought to exploit DNA-protein binding for nanomachines, particularly thrombin binding aptamers (TBA). Due to the high specificity and reversibility of binding, TBA and thrombin have been attractive for nanomachines that cycle through states by addition of $\mathrm{DNA}^{[1,2]}$. However, for biomolecular machines to be ultimately useful, they must be interfaced to synthetic surfaces to harness cumulative molecular-level changes and generate useful work as macroscopic motion, triggered assembly, or reporting a change ${ }^{[3-11]}$.

Unfortunately, control over the nanoscale biomolecular interface is severely lacking. "Wiring" biomolecules onto surfaces without hindering the designed behavior is challenging. DNA and proteins have strong tendencies to denature on surfaces due to the numerous noncovalent interactions between the biomolecule and the surface and coating molecules, and denaturation is influenced by a myriad of parameters including surface material, coating chemistry, and curvature ${ }^{[12-16]}$. Such interactions can accumulate and result in drastic deviations from solution behavior, affecting binding affinity, kinetics and yield, ${ }^{[12,13,17]}$ sometimes

resulting in unexpectedly improved situations ${ }^{[4,18]}$. Approaches to remove interface effects have 
met limited success ${ }^{[19,20]}$, where ligands thought to be inert exhibit unexpected stickiness. These issues present a huge challenge for not only nanobiomachines, but nearly every application with surface immobilized biomolecules ${ }^{[21,22]}$.

Despite broad interest in using NPs coupled to biomolecules, systematic characterization of interface biomolecular nanomachinery has been lacking. For example, it is known that TBA binding to thrombin is influenced by the properties of the surface it is on as well as its molecular neighbors, which can give rise to intermolecular repulsion that hinder its ability to fold and bind. However, these effects have been described only qualitatively, as there are no direct probes. A parameter describing these interface interactions would not only yield a deeper understanding of surface effects but also enable prediction of molecular behavior. Furthermore, it would allow comparison, which would be beneficial especially for surface chemistry modification strategies, which can drastically affect biomolecular behavior and are notorious for being difficult to control. Most investigations to date have not been quantitative, but instead phenomenological, probing only whether or not biomolecules adsorb. Unfortunately, these empirical observations cannot capture quantitative information, and therefore are insufficient. Thus, a map of TBA binding regimes as a function of surface properties would greatly enhance the ability to construct interfaces to biomolecules. A few such studies on planar surfaces exist ${ }^{[3,4,23]}$ but to the best of our knowledge, quantitative studies for NPs are few ${ }^{[24]}$, despite the fact that the surface dominates on the nanoscale.

Here we develop a new thermodynamic treatment for quantifying TBA nanomachinery on gold nanorods (NRs) interacting with thrombin. This thermodynamic treatment allows for quantitative description of the interface effects. We systematically probe how the interactions vary with TBA coverage, sequence, surface chemistry, and blocking proteins. We show that the 
term describing surface work is key for quantifying interface effects on TBA behavior. This

treatment is a promising framework to describe directed self-assembly of $\mathrm{NPs}^{[8,25]}$, and could be used to advance the state-of-the-art in organizing nanoscale 'building blocks' into assemblies, which have lagged behind advances in synthesis and characterization of NPs and macromolecules $^{[26]}$.

\section{Results and Discussion}

\subsection{Theoretical}

Consider the interaction of thrombin with TBA immobilized on NRs (Figure 1). Because thrombin binds specifically to TBA at exosite I (green) and exosite II (purple), binding results in aggregation of TBA decorated NRs (NR-TBA). From a thermodynamic perspective this can be represented as the equilibrium between a free (state I) and an aggregated state (state IV).

To describe this equilibrium system, we introduce the concept of unit block of aggregation, defined as an arbitrary fundamental complex from which the entire aggregate may be constructed by purely translational additions. For a NR-TBA-thrombin aggregate, several thrombins are bound to each NR-TBA. Thus, it is convenient to identify a NR-TBA bound to a convenient number $n$ of thrombins as the unit block. Consequently, equilibrium between states I and IV can be described as the binding equilibrium of $n$ thrombin molecules to a single NR-TBA, where NR-TBA acts as a receptor displaying $n$ binding sites with invariant identical affinities (e.g. a $n$ valent uniform receptor ${ }^{[27]}$ ). Within this scheme, state IV represents the particular case of the smallest aggregate with $n=1$ (Figure 1).

Therefore, aggregation can be described in terms of the binding efficiency for one TBA, $\theta^{[27]}$ 


$$
\theta=\frac{\Theta}{n}=\frac{K^{\sigma}[T h]}{1+K^{\sigma}[T h]}
$$

where $\Theta$ is the binding efficiency of the NR-TBA receptor as a whole, $K^{*}$ is the equilibrium association constant of the TBA-thrombin binding confined at the NR surface and [Th] is the equilibrium concentration of thrombin in solution.

TBA-thrombin binding is confined on the NR surface, so the overall Gibbs free energy of the reaction is comprised of both chemical and surface contributions. The chemical contribution is related to TBA-thrombin binding, whereas the surface contribution describes the work spent in accommodating the thrombin on the surface and the concomitant nanoscale rearrangement of bound and adjacent TBA molecules ${ }^{[4,5]}$. The overall surface effect on aggregation can be understood if we construct a thermodynamic cycle that goes from the free state (state I) to the aggregated state (state IV), broken down into three hypothetical steps (Figure 1). The first step describes release of TBA from the NR to the solution (I to II), the second step TBA-thrombin recognition in solution (II to III), and the third conjugation of the TBA-thrombin complexes to the NR (III to IV).

Each step is characterized by a standard molar Gibbs free energy: release of TBA from the NR, $\Delta_{r} G_{0}{ }^{r e l}$, binding of TBA to thrombin, $\Delta_{r} G_{0}{ }^{\text {bind }}$ and re-conjugation of TBA-thrombin to the $\mathrm{NR}, \Delta_{r} G_{0}{ }^{c o n j}$. It follows that the standard molar Gibbs free energy of aggregation, $\Delta_{r} G_{0}{ }^{a g g}$, is given by

$$
\Delta_{r} G_{0}{ }^{a g g}=\Delta_{r} G_{0}{ }^{r e l}+\Delta_{r} G_{0}{ }^{b i n d}+\Delta_{r} G_{0}{ }^{c o n j}
$$

The surface work, $W^{s}$, can be defined by gathering the non-recognition contributions, or $W^{*}=$ $\Delta_{r} G_{0}{ }^{r e l}+\Delta_{r} G_{0}{ }^{\text {conj }}$. Thus $\Delta_{r} G_{0}{ }^{a g g}$ can be rewritten as

$$
\Delta_{r} G_{0}{ }^{a g g}=W^{\circ}+\Delta_{r} G_{0}{ }^{\text {ind }}
$$


which elucidates the energy of NR aggregation directed by molecular recognition confined on NR surfaces. In particular, it is not equal to the energy of molecular recognition free in solution, as it is usually assumed. The difference relies in the surface work component, $W^{*} . W^{o}$ can be positive or negative, and therefore obscure or promote aggregation. For example, a positive $W^{\circ}$ exceeding $\Delta_{r} G_{0}{ }^{\text {bind }}$ fully inhibits aggregation $\left(\Delta_{r} G_{0}{ }^{a g g}>0\right)$. The free energy of aggregation equals that of binding $\left(\Delta_{r} G_{0}{ }^{a g g} \cong \Delta_{r} G_{0}{ }^{\text {bind }}\right)$ only if $W^{\circ} \cong 0$, which occurs when the release and conjugation energies are similar or insignificant. These two cases can be described as "mild" surface conditions, possibly resulting from weak or negligible lateral interactions between surface confined species.

From the molecular machine standpoint, $W^{*}$ is co-operatively triggered by molecule-surface and molecule-molecule lateral interactions, such as hydration and thermal fluctuation forces, originating from nanoscale molecular motions fuelled by the interplay of surface molecular recognition, electrolyte surface density variation and "squeezing" between the NP surfaces ${ }^{[4,28]}$.

Equation (3) can be used to evaluate $W^{\circ}$ from dose-response data for the binding variables $\theta$ and [Th]. If we express $\Delta_{r} G_{0}{ }^{a g g}$ as $-R T \ln K^{o}$ and $\Delta_{r} G_{0}{ }^{b i n d}$ as $-R T \ln K^{b}$, where $K^{b}$ is the equilibrium association constant of TBA-thrombin in solution, we can rearrange Equation (3):

$$
\frac{1}{K^{\sigma}}=\frac{1}{K^{\mathrm{b}}} \exp \left(\frac{W^{\sigma}}{R T}\right)=K_{\mathrm{d}}^{\mathrm{b}} \exp \left(\frac{W^{\sigma}}{R T}\right)
$$

where $K_{d}^{b}$ is the TBA-thrombin dissociation constant in solution. Substituting Equation (4) into Equation (1) yields an expression that can quantify $W^{\circ}$ :

$$
\theta=\frac{1}{\frac{K_{\mathrm{d}}^{\mathrm{b}}}{[T h]} \exp \left(\frac{W^{\sigma}}{R T}\right)+1}
$$


Equation (5) can be interpreted as a Langmuir isotherm that specifically describes selfassembly of NPs directed by molecular interactions. It is related to the Stern equation (for describing electrolyte adsorption at a charged surface) and the modified Langmuir by Fowler and Guggenheim, implemented to take into account lateral interactions in adsorption of gases on solids. ${ }^{[29]}$ Here we ascribe a physical meaning to the modification via the thermodynamic cycle, i.e. by describing the energy of aggregation as a sum of surface and molecular recognition contributions. Note that when $W^{\circ}=0$, Eqn. 5 reduces to the Langmuir isotherm, which does not account for intermolecular interactions.

\subsection{Synthesis and characterization of TBA decorated NRs}

We deployed this thermodynamic framework on NR-TBA with different surface treatments (Figure 2a). NRs were monodisperse in size, as confirmed by TEM (Figure 2b), with a length of $35 \pm 5 \mathrm{~nm}$ and width of $10 \pm 1 \mathrm{~nm}$ (aspect ratio $=3.6 \pm 0.7)$. TBAs used were terminated with a 5'SH that allowed conjugation to the gold NRs. A 15 thymine $\left(T_{15}\right)$ spacer was either present ( $\mathrm{T}_{15}$-TBA) or not (TBA). TBA surface density for $\mathrm{NR}-\mathrm{T}_{15}$-TBA was varied by tuning the NR:TBA incubation ratio during conjugation, resulting in coverages of $5.6 \pm 1.1 \times 10^{12} \mathrm{TBA} / \mathrm{cm}^{2}$ (low coverage) vs. $12.3 \pm 2.5 \times 10^{12} \mathrm{TBA} / \mathrm{cm}^{2}$ (high coverage). The NR surface chemistries were also varied. NR-T 15 -TBA were "backfilled" with ligands of thiolated polyethylene glycol (mPEG), which is used extensively as a surface coating ligand for NPs to prevent fouling and non-specific adsorption. Since PEGylation of NP surfaces sometimes does not remove nonspecific adsorption $^{[30,31]}$, we probed PEGylation under both strong and mild conditions, with incubation ratios of 5:1 and 0.5:1 mPEG: $\mathrm{T}_{15}$-TBA, respectively. Finally, the effect of "blocking" the surface was investigated with human serum albumin (HSA, $0.3 \mathrm{w} / \mathrm{v} \%$ ), a protein widely used 
to prevent aggregation and non-specific adsorption for both NP and planar surfaces (For details, see Experimental Section).

Gel electrophoresis was used to probe the effect of the different surface conditions on the NRs (Figure 2c). Conjugation of the NRs to the $\mathrm{T}_{15}$-TBA (Lane 3) and to TBA (Lane 2) was confirmed by a shift to lower mobility relative to MHA coated NRs (Lane 5). Decreasing the DNA:NR incubation ratio resulted in a smaller mobility shift (Lane 4), confirming the lower coverage of the $\mathrm{T}_{15}$-TBA on the NR surface. This was independently confirmed by quantifying the DNA on the NR surface by chemical displacement (see Experimental Section) ${ }^{[32,33]}$. Incubating the NR- $\mathrm{T}_{15}$-TBA with HSA also resulted in a shift to lower mobility (Lane 1), due to the increase in NR- $\mathrm{T}_{15}$-TBA size upon adsorption of the HSA, confirming successful blocking. Incubation of NR- $\mathrm{T}_{15}$-TBA (high coverage) with $\mathrm{mPEG}(\mathrm{MW}=5000)$ at a 5:1 ratio resulted in further shifts to lower mobility (Lane 7) relative to NR- $\mathrm{T}_{15}$-TBA high coverage (Lane 6), indicating that some of the neutral mPEG was put onto the surface of the NR- $\mathrm{T}_{15}$-TBA. However, under mild PEGylation conditions (0.5:1, Lane 9), no mobility shift was observed relative to NR- $\mathrm{T}_{15}$-TBA high coverage (Lane 8 ).

\subsection{Probing thrombin directed self-assembly}

The TBA studied was a single-stranded DNA 15mer 5'-GGTTGGTGTGGTTGG-3' [34]. When folded into a structure stabilized by two G-quartets (Figure 1) ${ }^{[35]}$, it binds strongly to thrombin at either exosite I or exosite II in a manner that is highly specific and reversible, with a binding constant $K_{D}$ that ranges from 1-100's of nM. Because thrombin can bind to two TBAs $^{[35]}$, NR-TBA will self-assemble upon binding to thrombin (Figure 1). This results in a red shift and broadening of the NR surface plasmon resonance (SPR) peak. Figure 3a shows the absorption spectrum of NR functionalized with $\mathrm{T}_{15}$-TBA incubated with $121 \mathrm{nM}$ of thrombin as 
a function of time. The longitudinal SPR red shifts and broadens with time, suggesting aggregation. To quantify aggregation, the ratio of extinctions at $400 \mathrm{~nm}$ and $800 \mathrm{~nm}\left(\mathrm{~A}_{400} / \mathrm{A}_{800}\right)$ is plotted, which increases as a function of time in the presence of thrombin (Figure 3b). $\mathrm{A}_{400} / \mathrm{A}_{800}$ time courses are collected for several thrombin concentrations ( $0-121 \mathrm{nM}$, Figure $3 \mathrm{~b})$. The $\mathrm{A}_{400} / \mathrm{A}_{800}$ endpoint at $\mathrm{t}=1 \mathrm{hr}$ is used to define when equilibrium is attained, and is plotted as a function of thrombin concentration (Figure 3c) to construct an aggregation dose-response curve, or isotherm. The aggregation isotherm exhibits Langmuir-like behavior, where after a threshold it increases with increasing thrombin concentration, eventually saturating.

In order to prove that the self-assembly is primarily due to the specific interaction between thrombin and the TBA on the NR, we performed a competitive inhibition. Already formed NR$\mathrm{T}_{15}$-TBA -thrombin aggregates were incubated with a DNA strand complementary to the TBA. Because TBA must maintain a G-quartet structure for binding, hybridization to a complement will not permit it to self-fold and bind to thrombin. Upon addition of an excess of the TBA complement (4340:1 complement: TBA on the NR surface) to the $38.4 \mathrm{nM}$ thrombin-aggregated sample (Figure 3d, gray), $\mathrm{A}_{400} / \mathrm{A}_{800}$ decreases to 1.0, indicating that the aggregate was disrupted, thus confirming that self-assembly is due to the specific TBA-thrombin interaction. In addition, disruption of the aggregate by heat was also probed. Already formed aggregates with a thrombin concentration of $121 \mathrm{nM}$ were exposed to a heating-cooling cycle and monitored by tracking $\mathrm{A}_{400} / \mathrm{A}_{800}$ (Figure 3f). As the temperature was increased, $\mathrm{A}_{400} / \mathrm{A}_{800}$ decreased with a sigmoidal drop centered at $\sim 42{ }^{\circ} \mathrm{C}$, suggesting that the aggregate was melted by denaturation of the TBA or the thrombin. Decreasing the temperature from $80{ }^{\circ} \mathrm{C}$ down to $25^{\circ} \mathrm{C}$ did not result in a change in $\mathrm{A}_{400} / \mathrm{A}_{800}$, suggesting that the aggregate could not form again. This could be due to the fact that 
the thrombin was irreversibly denatured ${ }^{[36]}$. These experiments further confirm that the aggregation is due to the specific interaction of thrombin with the TBA on the NR surface.

\subsection{Nanomachinery of TBA on NRs}

Aggregation isotherms were analyzed using a modified Langmuir based on Equation 5, assuming that $\mathrm{A}_{400} / \mathrm{A}_{800}$ was a measure of the number of aggregated NR-TBA ${ }^{[37]}$, and thus of the TBA-thrombin surface binding efficiency $\theta$. Data points from three experimental replicates were fitted by a best-fit procedure to obtain $\theta$ and $W^{o}$, with associated errors evaluated.

Aggregation as a function of $[T h]$ and fits are reported (Figures $4 \mathrm{a}-4 \mathrm{~g}$ ). The fits are compared in Figure 4d. High and low coverage NR-T 15 -TBA display isotherms that differ in two ways (Figures $4 \mathrm{a}$ and $\mathrm{b}$ ). First, the aggregation onset, or the threshold thrombin concentration, $[T h]_{\mathrm{T}}$, for the high coverage sample is $\sim 30 \mathrm{nM}$, while it is $\sim 15 \mathrm{nM}$ for low coverage. Also, the rise in the curve is steeper for high coverage NR- $\mathrm{T}_{15}-\mathrm{TBA}$. This indicates that changing TBA surface coverage affects thrombin binding to NR- $\mathrm{T}_{15}$-TBA. Treating high coverage NR- $\mathrm{T}_{15}$-TBA surface with mild mPEG conditions also affected the isotherm, resulting in a steeper curve rise and highest $[T h]_{\mathrm{T}}$ at $\sim 35 \mathrm{nM}($ Figure $4 \mathrm{c})$. On the contrary, no isotherm was registered for NR- $\mathrm{T}_{15^{-}}$ TBA exposed to stronger PEGylation (Figure 4g), showing that aggregation varies with surface modification conditions. NRs coated with TBA lacking $\mathrm{T}_{15}$ exhibited no aggregation (Figure $4 \mathrm{e}$ ), suggesting that TBA on the NR was unable to bind thrombin. TBA coverage was $28.6 \pm 5.8 \times$ $10^{12} \mathrm{TBA} / \mathrm{cm}^{2}$ as determined by chemical displacement, which exceeds a monolayer and is likely

due to formation of intermolecular G-quartets ${ }^{[38]}$ that would disrupt the ability to bind thrombin. Incubation of high coverage NR- $\mathrm{T}_{15}$-TBA with HSA prevented thrombin binding and thus aggregation, as indicated by negligible change in $\mathrm{A}_{400} / \mathrm{A}_{800}$ (Figure $4 \mathrm{f}$ ). 
The thermodynamic model was used to map how $W^{*}$ changes as a function of $\theta$ and $[T h]_{\mathrm{T}}$ for the samples that aggregate, as a function of TBA coverage and surface chemistry (Figure 5). First, values for $W^{*}$ are all negative and $\sim-10 \mathrm{~kJ} / \mathrm{mol}$, indicating that $W^{*}$ significantly participates along with TBA-thrombin recognition in driving aggregation. From Eq. (3) and taking into account that thrombin-TBA recognition energy is $\Delta_{r} G_{0}{ }^{\text {bind }} \sim-37 \mathrm{~kJ} / \mathrm{mol}{ }^{[39]}$, it follows that nearly $30 \%$ of the aggregation energy, $\Delta_{r} G_{0}{ }^{a g g}$, that results $\sim-50 \mathrm{~kJ} / \mathrm{mol}$, comes from $W^{\circ}$. This shows that aggregation is not due solely to TBA-thrombin binding, as it is predominantly assumed.

From the same calculation we learn that $\Delta_{r} G_{0}{ }^{a g g}$ corresponds to $\sim 20 k_{\mathrm{B}} T$, confirming that NRTBA aggregation is long-lived, on the timescale of hours or days ${ }^{[28]}$. Conversely, $W^{*}$ values in $k_{\mathrm{B}} T$ units are $3.2 \pm 0.5 k_{\mathrm{B}} T, 4.3 \pm 0.6 k_{\mathrm{B}} T$ and $5.0 \pm 1.1 k_{\mathrm{B}} T$ for low and high coverage NR-TBA and mPEG backfilled NR-TBA, respectively (Figure 5a, top axis). These values are high enough to say that the in-plane intermolecular interactions underpinning $W^{*}$ are significantly larger than thermal vibrational energy $k_{\mathrm{B}} T$. This suggests that the in-plane interaction lifetime is $<1 \mathrm{sec}$ ${ }^{[28]}$ and that the timescale of TBA motion is well below the timescale of NR-TBA aggregation. Obtained $\theta$ for NR- $\mathrm{T}_{15}$-TBA high and low coverage and $\mathrm{mPEG}$-backfill are identical within experimental errors and close to $100 \%$ (Figure 5a). This means that aggregation was nearly complete for all samples. However, the $W^{\circ}$ values differ, where low coverage has the lowest absolute value, $-8.0 \pm 1.3 \mathrm{~kJ} / \mathrm{mol}$ (triangle), higher coverage is higher, $-10.7 \pm 1.5 \mathrm{~kJ} / \mathrm{mol}$ (square), and mPEG under mild conditions results in the highest value, $-12.4 \pm 2.8 \mathrm{~kJ} / \mathrm{mol}$ (circle). Evidently, increasing TBA surface density increases its absolute $W^{s},|W|$. This could be due to the fact that for aggregation, more $\left|W^{\circ}\right|$ is done to accommodate thrombin on crowded surfaces. Furthermore, introduction of small amounts of mPEG, a bulky molecule, increases $\left|W^{\circ}\right|$. 
This difference in energy is taken from thrombin in solution, which gives rise to differences in $[T h]_{\mathrm{T}}$ (i.e. the thrombin chemical potential). $[T h]_{\mathrm{T}}$ required for the aggregation onset increases with increasing $\left|W^{\circ}\right|$, showing that samples with lower $\left|W^{\circ}\right|$ and thus smaller interface effects do not need as much thrombin to aggregate (Figure 5b).

These results show that TBA behavior can vary with varying coverage on the NR or NR surface chemistry, and in a way that does not affect its aggregation efficiency $\theta$. Conversely, this means that simply probing NR aggregation yields incomplete information on biomolecular behavior on the NR surface. Surprisingly, in view of $W^{*}$ data and Eq. (3) the NR- $T_{15}$-TBA sample with mild PEGylation has the highest energy of aggregation, $\Delta_{r} G_{0}{ }^{a g g}$, and thus forms the most stable aggregate. Even though the surface treatment does not yield a detectable amount of PEG on the NR by electrophoresis, its $W^{*}$ is distinct from high coverage NR- $\mathrm{T}_{15}-\mathrm{TBA}$. This shows that mild surface treatments that are difficult to detect can still result in significant $W^{\circ}$ changes. It also shows that PEGylation does not always result in inert surfaces that do not foul or experience non-specific adsorption, and highlights the variability and complexity of the interface.

$W^{\circ}$ can be expressed in $\mathrm{mN} / \mathrm{m}$ for comparison to $W^{\circ}$ of biomolecular transformations such as DNA hybridization, molecular recognition interactions ${ }^{[7]}$, and $\mathrm{pH}$ or electrolyte driven conformational changes confined to planar surfaces ${ }^{[3,4,23]}$. To the best of our knowledge, this is the first time analogous studies are reported for NPs. $W^{*}$ from MC experiments range from 1 to 10 's of $\mathrm{mN} / \mathrm{m}$, where an aptamer-protein study reports $W^{\circ}=9.6 \mathrm{mN} / \mathrm{m}^{[40]}$. By dividing obtained $W^{\circ}$ for low and high coverage NR- $\mathrm{T}_{15}$-TBA for the respective TBA surface coverage (see ${ }^{[4]}$ for a justification) we obtain $W^{\circ}=-0.74 \pm 0.26 \mathrm{mN} / \mathrm{m}$ and $W^{\circ}=-2.18 \pm 0.76 \mathrm{mN} / \mathrm{m}$, respectively, which are consistent with reported ranges. This suggests that $W^{\circ}$ involved in transformations of 
NP conjugated biomolecules is comparable to $W^{\circ}$ of biomolecules on planar surfaces.

Furthermore, for an average coverage of $10^{13} \mathrm{TBA} / \mathrm{cm}^{2}, 1$ TBA molecule occupies $\sim 10 \mathrm{~nm}^{2}$, corresponding to an area $\sim 3.5 \mathrm{~nm}$ diameter. By taking into account that a folded TBA molecule has an average size of $2.5 \mathrm{~nm}$, the average distance between TBAs is $\sim 1 \mathrm{~nm}$, which multiplied to $W^{*}$ gives a force on the order of $\mathrm{pN}$. Remarkably, these values are consistent with unfolding forces from single-molecule experiments ${ }^{[4]]}$.

\section{Conclusions}

We have introduced a thermodynamic framework that describes and quantifies interactions of TBA on NR surfaces with thrombin. The model relies on separating TBA-thrombin recognition energy from surface work, $W^{s}$, unique to confinement of the recognition event within NR surfaces. $W^{*}$ enables quantitative understanding of interfacial nanoscale phenomena "fuelled" by thrombin directed TBA-NR aggregation, and thus has significant ramifications for nanomachines which rely on extracting work from molecular movements.

In particular, $W^{\circ}$ results from accumulation of in-plane molecular forces of $\mathrm{pN}$ magnitude and lifetime $<1$ second. In the studied system $W^{\circ}$ is $\sim-10 \mathrm{~kJ} / \mathrm{mol}$ and substantially promotes aggregation. However, this is not a general rule, as $W^{\cdot}$ may turn out to be positive, which could potentially work against desired effects in some applications. Finally, $W^{*}$ is effective in mapping aggregation regimes as a function of different nanoparticle surface conditions.

The presented approach is broadly applicable to behavior of any NP confined ligand-receptor recognition and could significantly contribute to understanding surface confined biomolecular transformations, as it extends to nanoparticles quantitative information that currently could be accessed only for planar surfaces. 


\section{Experimental Section}

\section{Synthesis and characterization of the TBA decorated gold NRs}

Gold nanorods (NRs) were synthesized using a single surfactant non-seed-mediated growth method in $200 \mathrm{~mL}$ batches ${ }^{[42-44]}$. Briefly, gold chloride trihydrate $\left(\mathrm{HAuCl}_{4}\right)$, silver nitrate $\left(\mathrm{AgNO}_{3}\right)$, ascorbic acid $(\mathrm{AA})$ and sodium borohydride $\left(\mathrm{NaBH}_{4}\right)$ were added to a mixture of cetyltrimethylammonium bromide $(\mathrm{CTAB})$ and sodium chloride $(\mathrm{NaCl})$. The solution sat on the bench undisturbed overnight, during which time it turned reddish brown, indicating the presence of NRs. TEM analysis showed that the NRs had dimensions of $35 \pm 5 \mathrm{~nm} \times 10 \pm 1 \mathrm{~nm}$. All the reagents were purchased from Sigma Aldrich, except for $\mathrm{NaCl}$, that was from Mallinckrodt.

The CTAB surfactant on the NR surface was exchanged with mercaptohexanoic acid (MHA) by a round-trip ligand exchange ${ }^{[45]}$; this method involves transferring the NR to an organic phase and then back to an aqueous phase with mercaptohexanoic acid (MHA) as the ligand.

In order to conjugate the NR-MHA with DNA, 6nM NR were incubated with DNA in 10 $\mathrm{mM}$ phosphate buffer through a salt aging process ${ }^{[46,47]}$. The DNA oligonucleotides were purchased from Integrated DNA Technologies fluorescently labeled at the 3' ends with tetramethylrhodamine (TMR), and thiolated in the 5' ends. The sequences used were 5'-SHTTTTTTTTTTTTTTTGGTTGGTGTGGTTGG-TMR-3' (T ${ }_{15}$-TBA) and 5'-SHGGTTGGTGTGGTTGG-TMR 3' (TBA). The DNA:NR ratios were 1000:1 DNA/NR for the high coverage particles and 200:1 DNA/NR for the low coverage particles.

DNA conjugation to the NR was confirmed by mobility changes of the NR-DNA in gel electrophoresis with $0.5 \%$ agarose gels in $0.5 \times$ TBE. DNA per NR was quantified by chemical displacement ${ }^{[32]}$ with $1 \mathrm{mM}$ mercaptohexanol $(\mathrm{MCH})$. Free DNA was quantified via fluorescence 
spectroscopy. High coverage yielded $12.3 \pm 2.5 \times 10^{12} \mathrm{~T}_{15}$-TBA $/ \mathrm{cm}^{2}$, and low coverage yielded $5.6 \pm 1.1 \times 10^{12} \mathrm{~T}_{15}-\mathrm{TBA} / \mathrm{cm}^{2} . \mathrm{TBA}$ coverage was $28.6 \pm 5.8 \times 10^{12} \mathrm{TBA} / \mathrm{cm}^{2}$.

Further surface modifications were performed on the NR- $\mathrm{T}_{15}$-TBA. HSA block was achieved after incubating a $0.17 \mathrm{nM}$ solution of the NR- $\mathrm{T}_{15}$-TBA in $0.3 \%(\mathrm{w} / \mathrm{v}) \mathrm{HSA}$. Also, NR- $\mathrm{T}_{15}$-TBA were $\mathrm{mPEG}$ backfilled by overnight incubation of the with $\mathrm{mPEG}$ at a ratio of 5:1 and 0.5:1 mPEG:TBA on the NR surface. mPEG backfill was monitored by electrophoretic mobility changes of the NR- T $_{15}$-TBA-mPEG, where the 5:1 mPEG;DNA backfill showed a mobility shift, but the $0.5: 1$ did not.

\section{Monitoring the NR-thrombin interaction by optical absorption}

$100 \mu 1$ of $0.17 \mathrm{nM}$ DNA conjugated NR were mixed with $50 \mu \mathrm{l}$ of thrombin in a PBS solution with $0.1 \%$ BSA. After the addition of thrombin, the extinction of the NR was monitored for an hour. Aggregation of the NR was calculated by dividing the $400 \mathrm{~nm}$ extinction $\left(\mathrm{A}_{400}\right)$ by the $800 \mathrm{~nm}$ extinction $\left(\mathrm{A}_{800}\right)$ at each time point. The aggregation was normalized by dividing every value by the aggregation without thrombin addition.

In order to ensure that the interaction between the TBA and the thrombin was reversible, we heated the aggregated sample from 25 to $80^{\circ} \mathrm{C}$ and measured its absorbance. Moreover, we tested that the interaction was reversible by adding the complementary DNA; purchased from Integrated DNA technologies with a sequence 5'-CCAACCACACCAACC-3', to $150 \mu \mathrm{L}$ of the aggregated sample, and monitoring the absorbance for four hours.

\section{Fitting of aggregation isotherms}

The first step to exploit Equation (5) to fit the experimental curves consists in expressing the binding efficiency, $\theta$, in terms of the signal intensity, $I$. Since $\theta$ and $I$ are proportional, we can set $\theta=I \cdot \beta^{-1}$, in which $\beta$ is the proportional constant. We obtain 


$$
I=\frac{\beta}{\frac{K_{d}^{b}}{[T h]} \exp ^{\frac{W^{\sigma}}{R T}}+1}
$$

However, this Langmuir equation fails to fit the experimental data and it needs to be further optimized. By using the mathematical principle of translation, we applied a rigid shift of the Equation (6) with respect to the $x$ and $y$ axes in order to take into account the onset of aggregation. These shifts were subtracted from the equilibrium concentration and from the signal intensity to give the following equation

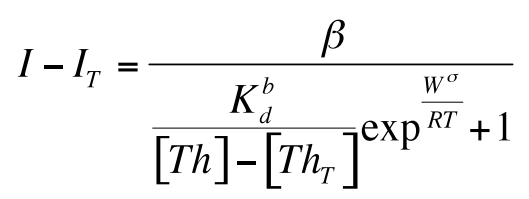

In which $I_{T}$ and $T h_{T}$ refer to the values of signal intensity and equilibrium concentration at the threshold. $K_{d}^{b}$ is the TBA-thrombin dissociation constant in homogeneous solution fixed at $3.3 \pm$ $1.1 \times 10^{-7} \mathrm{M}^{[39]} \cdot b$ and $W^{s}$ are the free parameters of the fitting.

Data are fitted using Equation (6) over three repetitions of the same experiments and the errors on the free parameters are assigned as a result of the fitting algorithm (with 95\% confidence bounds).

Finally, we described the NR aggregation process through a single binding of NR confined TBA and thrombin (Equation 1). Since aggregation was conducted in excess of thrombin with respect to NR concentration (about $6 \mathrm{nM}$ ), we may assume in first approximation that the concentration of thrombin not involved in that binding event (i.e. formally in solution), Th, does not change significantly before and after aggregation $([T h] \equiv T h)$. 


\section{Acknowledgements}

We thank the MIT-Cariplo Exchange program. H. d. P. G. was supported by the MIT IQS exchange program. We thank Zhichuan Xu for TEM imaging and the Center for Materials Science and Engineering for use of the TEM. We thank the Jones Prather and Van Vliet groups for use of equipment. P. B. and S. F. wish to thank Daniele Maiolo, Ivano Alessandri, Laura E. Depero for fruitful discussions and suggestions.

\section{Figure Captions}

Figure 1. Thermodynamic cycle describing the aggregation of NR-TBA in the presence of thrombin.

Figure 2. a) NR samples probed, b) TEM image of NRs, c) gel electrophoresis of NR samples probed. Lane 1: NRs conjugated to $\mathrm{T}_{15}$-TBA with high coverage exposed to HSA, Lane 2: NRs conjugated to TBA, Lane 3: NRs conjugated to $\mathrm{T}_{15}$-TBA with high coverage, Lane 4: NRs conjugated to $\mathrm{T}_{15}$-TBA with low coverage, Lane 5: MHA coated NRs before conjugation to DNA. Lane 6: NRs conjugated to $\mathrm{T}_{15}$-TBA with high coverage, Lane 7: NRs conjugated to $\mathrm{T}_{15^{-}}$ TBA with high coverage and exposed to mPEG (MW 5000) at 5:1 mPEG:TBA, Lane 8: NRs conjugated to $\mathrm{T}_{15}$-TBA with high coverage, Lane 9: NRs conjugated to $\mathrm{T}_{15}$-TBA with high coverage and exposed to mPEG (MW 5000) at 0.5:1 mPEG:TBA.

Figure 3. a) Change in absorption spectra of NR-T 15 -TBA high coverage as a function of time upon incubation with $121 \mathrm{nM}$ of thrombin (arrow indicates increasing time), b) $\mathrm{A}_{400} / \mathrm{A}_{800}$ as a function of time for NR-T 15 -TBA high coverage for different thrombin concentrations, c) aggregation isotherm for NR- $\mathrm{T}_{15}$-TBA high coverage, d) $\mathrm{A}_{400} / \mathrm{A}_{800}$ as a function of time upon incubation with thrombin $(\mathrm{t}=0 \mathrm{sec})$ and complementary DNA strand (gray area, $50 \mathrm{~s}), \mathrm{e}$ ) 
$\mathrm{A}_{400} / \mathrm{A}_{800}$ of already formed NR- $\mathrm{T}_{15}$-TBA -thrombin aggregates as a function of changing the temperature from $25^{\circ} \mathrm{C}$ to $80^{\circ} \mathrm{C}$ and back down to $25^{\circ} \mathrm{C}$ (arrows indicate direction).

Figure 4. Modified Langmuir aggregation isotherms for a) NR- $\mathrm{T}_{15}$-TBA high coverage, $\mathrm{b}$ ) NR-T 15 -TBA low coverage, c) NR-T 15 -TBA mPEG backfill 0.5:1 mPEG:TBA, d) fits for plots ac, e) NR-TBA without poly $T$ spacer (the dashed line is the average value), f) $N R-T_{15}-T B A$ HSA block (the dashed line is the average value), g) NR- $\mathrm{T}_{15}$-TBA high coverage mPEG backfill 5:1 mPEG:TBA.

Figure 5. a) $\theta$ vs. - $W^{\circ}$ for NR- $\mathrm{T}_{15}$-TBA high coverage (square), NR-T 15 -TBA low coverage (triangle), and NR-T 15 -TBA -PEG backfill 0.5:1 (circle), b) $T h_{\mathrm{T}}$ vs. $-W^{\circ}$ for NR- $\mathrm{T}_{15}$-TBA high coverage (square), NR- $\mathrm{T}_{15}$-TBA low coverage (triangle), and NR- $\mathrm{T}_{15}$-TBA -mPEG backfill $0.5: 1$ (circle).

\section{References}

[1] S. Beyer, F. C. Simmel, Nucleic Acids Res. 2006, 34, 1581.

[2] W. U. Dittmer, A. Reuter, F. C. Simmel, Angew. Chem. 2004, 43, 3550.

[3] W. Shu, D. Liu, M. Watari, C. K. Riener, T. Strunz, M. E. Welland, S. Balasubramanian, R. A. McKendry, J. Am. Chem. Soc. 2005, 127, 17054.

[4] S. Federici, G. Oliviero, K. Hamad-Schifferli, P. Bergese, Nanoscale 2010, 2, 2570.

[5] P. Bergese, G. Oliviero, I. Alessandri, L. E. Depero, J. Colloid Interface Sci. 2007, 316, 1017.

[6] U. Feldkamp, C. M. Niemeyer, Angew. Chem. 2006, 45, 1856.

[7] J. Fritz, Analyst 2008, 133, 855. 
[8] M. Grzelczak, J. Vermant, E. M. Furst, L. M. Liz-Marzan, ACS Nano 2010, 4, 3591.

[9] C. M. Niemeyer, Angew. Chem. 2001, 40, 4128.

[10] R. Shenhar, V. M. Rotello, Acc. Chem.. Res. 2003, 36, 549.

[11] M. V. Yigit, D. Mazumdar, Y. Lu, Bioconjugate Chem. 2008, 19, 412.

[12] P. Gong, R. Levicky, Proc. Nat. Acad. Sci. 2008, 105, 5301.

[13] G. Oliviero, S. Federici, P. Colombi, P. Bergese, J. Mol. Recognit. 2011, 24, 182.

[14] A. A. Vertegel, R. W. Siegel, J. S. Dordick, Langmuir 2004, 20, 6800.

[15] M.-E. Aubin-Tam, K. Hamad-Schifferli, Langmuir 2005, 21, 12080.

[16] M.-E. Aubin-Tam, W. Hwang, K. Hamad-Schifferli, Proc. Nat. Acad. Sci. 2009, 106, 4095.

[17] E. V. Piletska, S. A. Piletsky, Langmuir 2010, 26, 3783.

[18] S. Park, K. Hamad-Schifferli, ACS Nano 2010, 4, 2555.

[19] W. Yang, L. Zhang, S. Wang, A. D. White, S. Jiang, Biomaterials 2009, 30, 5617.

[20] L. L. Rouhana, J. A. Jaber, J. B. Schlenoff, Langmuir 2007, 23, 12799.

[21] A. E. Nel, L. Madler, D. Velegol, T. Xia, E. M. V. Hoek, P. Somasundaran, F. Klaessig, V. Castranova, M. Thompson, Nature Mater. 2009, 8, 543.

[22] I. Lynch, T. Cedervall, M. Lundqvist, C. Cabaleiro-Lago, S. Linse, K. A. Dawson, Adv. Colloid Interface Sci. 2007, 134-135, 167.

[23] A. Valiaev, N. I. Abu-Lail, D. W. Lim, A. Chilkoti, S. Zauscher, Langmuir 2006, 23, 339.

[24] S. Connolly, S. Cobbe, D. Fitzmaurice, J. Phys. Chem. B 2001, 105, 2222.

[25] K. J. M. Bishop, C. E. Wilmer, S. Soh, B. A. Grzybowski, Small 2009, 5, 1600. 
[26] Y. Min, M. Akbulut, K. Kristiansen, Y. Golan, J. Israelachvili, Nature Mater. 2008, 7, 527.

[27] I. M. Klotz, Ligand-Receptor Energetics: A Guide for the Perplexed, Wiley, New York, 2007.

[28] D. Leckband, J. Israelachvili, Q. Rev. Biophys. 2001, 34, 105.

[29] A. W. Adamson, A. P. Gast, Physical Chemistry of Surfaces, Wiley, New York, 2000.

[30] E. E. Lees, M. J. Gunzburg, T.-L. Nguyen, G. J. Howlett, J. Rothacker, E. C. Nice, A. H. A. Clayton, P. Mulvaney, Nano Lett. 2008, 8, 2883.

[31] M.-E. Aubin-Tam, H. Zhou, K. Hamad-Schifferli, Soft Matter 2008, 4, 554.

[32] S. Park, K. A. Brown, K. Hamad-Schifferli, Nano Lett. 2004, 4, 1925.

[33] L. M. Demers, C. A. Mirkin, R. C. Mucic, R. A. Reynolds III, R. L. Letsinger, R. Elghanian, G. Viswanadham, Anal. Chem. 2000, 72, 5535.

[34] L. C. Bock, L. C. Griffin, J. A. Latham, E. H. Vermaas, J. J. Toole, Nature 1992, 355, 564.

[35] K. Padmanabhan, K. P. Padmanabhan, J. D. Ferrara, J. E. Sadler, A. Tulinsky, J. Biol. Chem. 1993, 268, 17651.

[36] S. Le Borgne, M. Graber, Appl. Biochem. Biotechnol. 1994, 48, 125.

[37] A. A. Lazarides, G. C. Schatz, J. Phys. Chem. B 1999, 104, 460.

[38] A.-J. Cheng, M. W. Van Dyke, Gene 1997, 197, 253.

[39] B. Pagano, L. Martino, A. Randazzo, C. Giancola, Biophys. J. 2008, 94, 562.

[40] C. A. Savran, S. M. Knudsen, A. D. Ellington, S. R. Manalis, Anal. Chem. 2004, 76, 3194. 
[41] C. Bustamante, Y. R. Chemla, N. R. Forde, D. Izhaky, Annu. Rev. Biochem. 2004, 73, 705.

[42] N. R. Jana, L. Gearheart, C. J. Murphy, Adv. Mater. 2001, 13, 1389.

[43] N. R. Jana, Small 2005, 1, 875.

[44] T. K. Sau, C. J. Murphy, Langmuir 2004, 20, 6414.

[45] A. Wijaya, K. Hamad-Schifferli, Langmuir 2008, 24, 9966.

[46] S. J. Hurst, A. K. R. Lytton-Jean, C. A. Mirkin, Anal. Chem. 2006, 78, 8313.

[47] Y. Zu, Z. Gao, Anal. Chem. 2009, 81, 8523. 


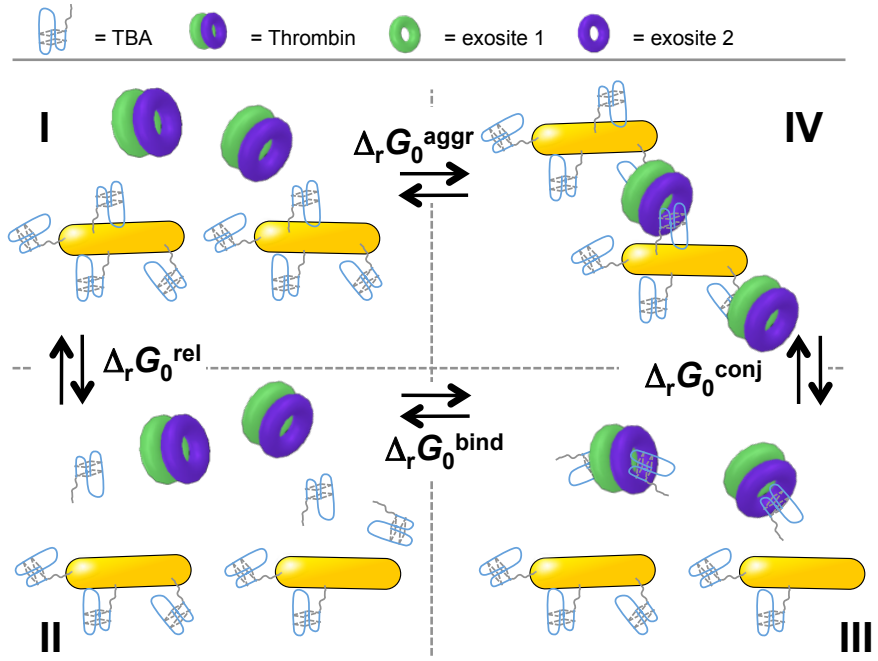

Figure 1 


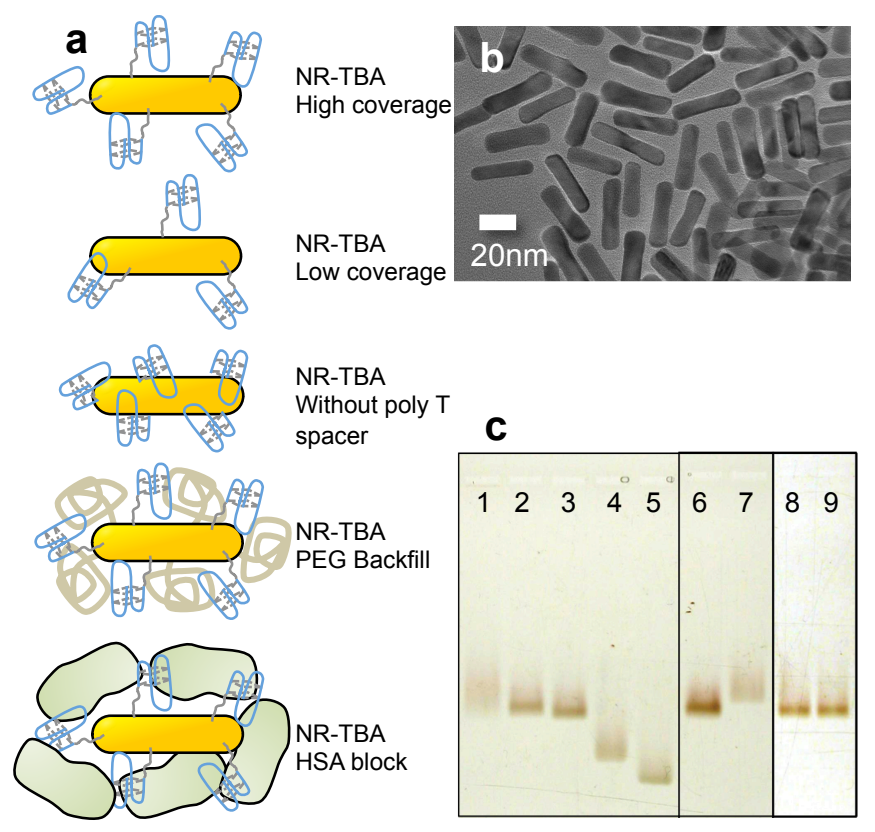

Figure 2 

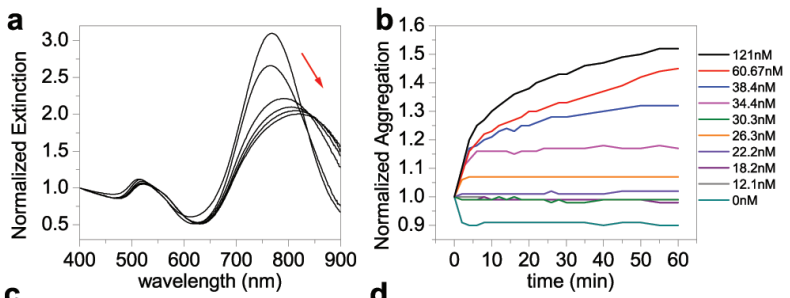

C
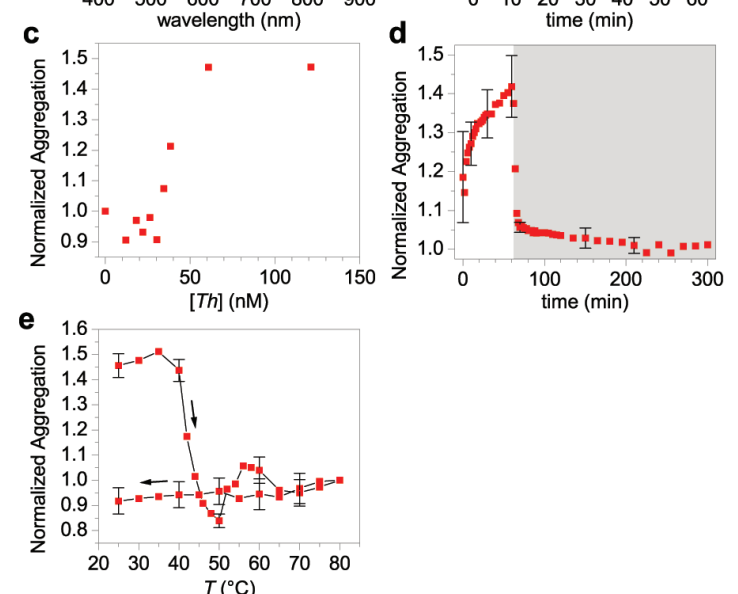

$\begin{array}{lll}60 & 70 \quad 80\end{array}$

$$
T\left({ }^{\circ} \mathrm{C}\right)
$$

Figure 3 

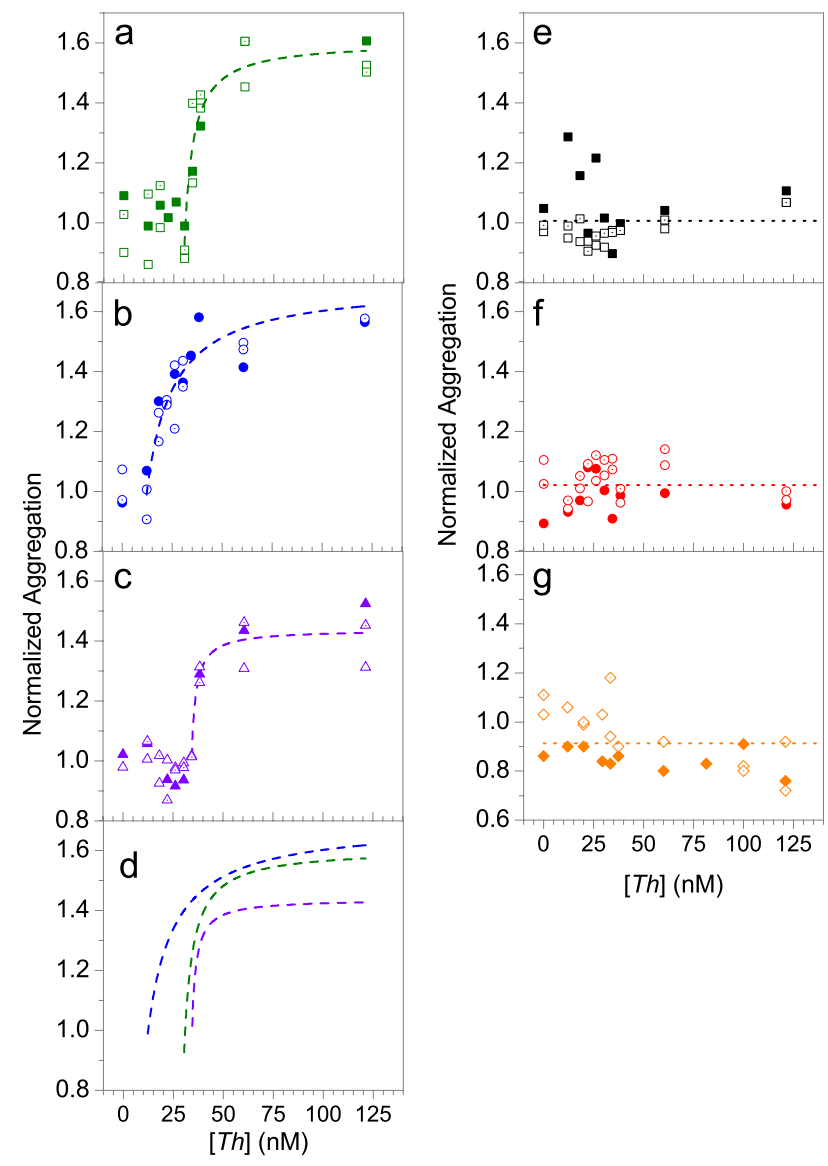

Figure 4 


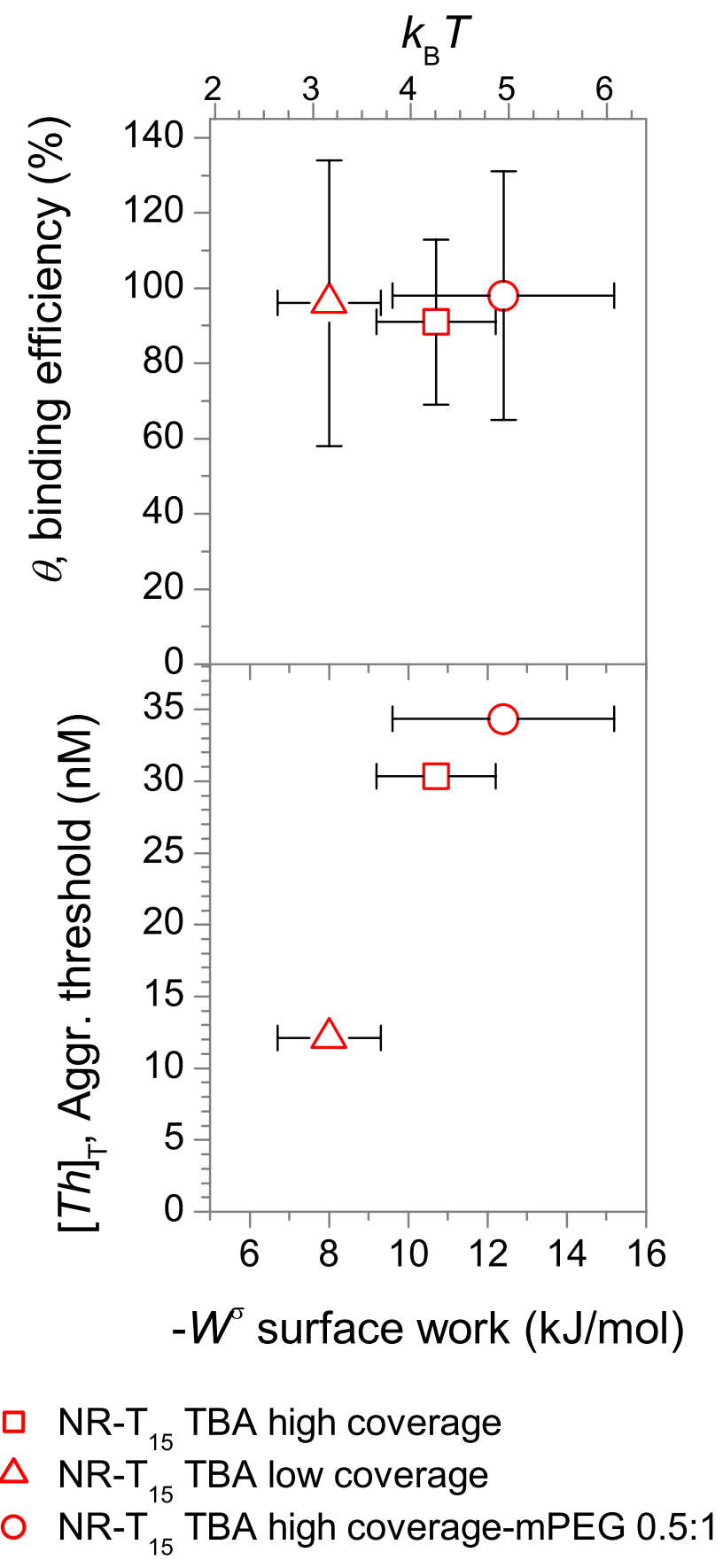

Figure 5 